\title{
Expression of UPR effector proteins ATF6 and XBP1 reduce colorectal cancer cell proliferation and stemness by activating PERK signaling
}

Claudia N. Spaan', Wouter L. Smit', Jooske F. van Lidth de Jeude', Bartolomeus J. Meijer', Vanesa Muncan', Gijs R. van den Brink ${ }^{1,3}$ and Jarom Heijmans ${ }^{1,2}$

\begin{abstract}
The unfolded protein response (UPR) acts through its downstream branches, PERK-elF2a signaling, IRE1aXBP1 signaling and ATF6 signaling. In the intestine, activation of the UPR through the kinase PERK results in differentiation of intestinal epithelial stem cells and colon cancer stem cells, whereas deletion of XBP1 results in increased stemness and adenomagenesis. How downstream activation of XBP1 and ATF6 influences intestinal stemness and proliferation remains largely unknown. We generated colorectal cancer cells (LS174T) that harbor doxycycline inducible expression of the active forms of either XBP1(s) or ATF6 ${ }^{1-373}$. Activation of either XBP1 or ATF6 resulted in reduced cellular proliferation and reduced expression of markers of intestinal epithelial stemness. Moreover, XBP1 and ATF6 activation reduced global protein synthesis and lowered the threshold for UPR activation. XBP1mediated loss of stemness and proliferation resulted from crossactivation of PERK-elF2a signaling and could be rescued by constitutive expression of elF2a phosphatase GADD34. We thus find that enforced activation of XBP1 and ATF6 results in reduction of stemness and proliferation. We expose a novel interaction between XBP1 and PERK-elF2a signaling.
\end{abstract}

\section{Introduction}

The intestinal epithelium is a rapidly renewing tissue that is fueled by intestinal stem cells located at the bottom of the crypt. Intestinal stem cells are defined by the capability to self-renew, proliferate, and differentiate and are therefore regarded as critical drivers that renew the intestinal epithelial layer every $3-5$ days ${ }^{1}$. Intestinal stem cells are not only important during homeostasis or after

\footnotetext{
Correspondence: Jarom Heijmans (j.heijmans@amsterdamumc.nl)

${ }^{1}$ Amsterdam UMC, University of Amsterdam, Department of Gastroenterology and Hepatology, Tytgat Institute for Liver and Intestinal Research, Meibergdreef 71, Amsterdam, The Netherlands

${ }^{2}$ Amsterdam UMC, University of Amsterdam, Department of Internal Medicine and Hematology, Meibergdreef 9, Amsterdam, The Netherlands

Full list of author information is available at the end of the article.

Effects of ATF6 and XBP1 on colon cancer cells

Edited by P. Agostinis
}

injury, but they are thought to be the cells of origin in the majority of colon cancers ${ }^{2}$. After acquiring consecutive mutations, mostly initiated by an inactivating mutation in the adenomatous polyposis coli $(A P C)$ gene, colon cancer stem cells arise from intestinal stem cells ${ }^{3,4}$. In contrast to differentiated tumor cells, these cancer stem cells are resistant to classical chemotherapy and novel strategies are emerging that aim at differentiating these cells in order to increase sensitivity to anticancer therapies ${ }^{5,6}$.

Previously, we found that intestinal stem cells differentiate upon activation of the unfolded protein response $(\mathrm{UPR})^{7}$. The UPR is an evolutionarily conserved signaling pathway activated upon endoplasmic reticulum (ER) stress, which occurs when unfolded and misfolded proteins accumulate in the lumen of the $\mathrm{ER}^{8,9}$. Activation of the UPR in the intestinal epithelium results in decreased

\section{(c) The Author(s) 2019}

(c) (i) Open Access This article is licensed under a Creative Commons Attribution 4.0 International License, which permits use, sharing, adaptation, distribution and reproduction cc. in any medium or format, as long as you give appropriate credit to the original author(s) and the source, provide a link to the Creative Commons license, and indicate if changes were made. The images or other third party material in this article are included in the article's Creative Commons license, unless indicated otherwise in a credit line to the material. If material is not included in the article's Creative Commons license and your intended use is not permitted by statutory regulation or exceeds the permitted use, you will need to obtain permission directly from the copyright holder. To view a copy of this license, visit http://creativecommons.org/licenses/by/4.0/. 
stemness. In addition, stem cells that are found in hyperproliferative crypts of mice that carry homozygous epithelial $A p c$ mutations were equally differentiated by UPR activation ${ }^{10}$. Moreover, ER stress decreases tumor burden in mice carrying the heterozygous APC mutation $^{11}$.

The UPR consists of three effector proteins: PKR like ER kinase (PERK), Inositol-requiring enzyme 1 (IRE1), and Activating Transcription Factor 6 (ATF6), which aim to resolve ER stress by transiently inhibiting global translation, expanding the ER capacity and upregulating important ER-resident chaperones, such as Grp78 and Grp94.

PERK is activated upon ER stress and is one of the four kinases that phosphorylates the alpha unit of eukaryotic translation initiation factor $2(\mathrm{eIF} 2 \alpha)^{13,14}$. The strong binding of phosphorylated eIF2 $\alpha$ to its GEF reduces levels of eIF2-GTP available for initiation of translation, resulting in reduced translation. Translation inhibition leads, among other things, to loss of cyclin D1 from cells, causing a G1 $\operatorname{arrest}^{15}$. Temporary inhibition of translation leads to a loss of short-lived proteins such as $M Y C^{16,17}$. Despite global inhibition of translation, a subset of mRNAs is selectively synthethized. These proteins include Activating transcription factor 4 (ATF4), C/EBP-homologous protein (CHOP) and Growth arrest and DNA damage-inducible 34 (GADD34), an eIF2 $\alpha$ phosphatase ${ }^{18}$. These mRNAs usually contain an upstream open reading frame.

IRE1 is a kinase and endoribonuclease that senses unfolded proteins in the lumen of the ER via its $\mathrm{N}$ terminal domain, which leads to activation by autophosphorylation. The endoribonuclease domain excises a 26-base-pair intron from the X-box-protein1 (XBP1) $m R N A$, which results in the $X B P 1(s) m R N A$, encoding an active transcription factor ${ }^{19}$. XBP1(s) activity leads to expansion of the ER and is crucial for cellular differentiation, especially in different secretory cell types ${ }^{20-24}$. Previously, it was shown that mice that lack epithelial Xbp1 have increased stem cell numbers and adenomagenesis. In this study, the oncogenic effects of loss of Xbp1 were attributed to Ire1 $\alpha$ and Stat3 signaling ${ }^{25}$.

ATF6 translocates to the Golgi apparatus upon ER stress. Site- 1 protease and Site- 2 protease cleave ATF6, whereupon the cytosolic transcription-factor is released $^{26,27}$. Expression of the active ATF6, ATF6 ${ }^{1-373}$, upregulates ER chaperones Grp78, and Grp94 and leads to expansion of the $\mathrm{ER}^{26,28}$.

We have previously shown that PERK-eIF2 $\alpha$ signaling plays an important role in the decrease of intestinal stemness after ER stress. Moreover, XBP1 has been linked to a decrease in intestinal stemness ${ }^{25}$. It is not known, however, to what extent ATF6 and XBP1(s) affect intestinal stemness ${ }^{29}$. Because XBP1 and ATF6 have extensively overlapping sets of target genes, we set out to investigate both XBP1 and ATF6 signaling in colon cancer cells, and investigate the crosstalk of these branches with PERK- eIF2 $\alpha^{20,29}$.

\section{Materials and methods \\ DNA constructs and cloning of plasmids}

$X B P 1(s)$ mRNA was amplified from a human cDNA pool using primers gatgcccagagaaccgtgaaagtg and cctcactttgtaatacactttcc. The truncated $A T F 6^{1-373}$ encoding the transcriptionally active part of ATF6 was a kind gift from Mori and coworkers ${ }^{26} . X B P 1(s)$ and $A T F 6^{1-373}$ were cloned into the pRetroTight vector (Clontech). The constitutively active hamster Gadd34 (also termed A1) was a kind gift from Ron and coworkers ${ }^{30}$. This coding sequence was subcloned into the pCDNA $3.1(+)$ vector (Invitrogen). Lentiviral shRNA constructs for PERK were obtained from the Mission shRNA library (Sigma).

\section{Cell culture and generation of stable cell lines}

LS174T colorectal cancer cells (ATCC CL188) and SW480 colorectal cancer cells (ATCC CCL228) were grown in Dulbecco's modified Eagle's medium with 10\% fetal calf serum (FCS) and 1\% penicillin/streptomycin under standard culture conditions. DLD-1 colorectal cancer cells (ATC CCL-221) were grown in RPMI with $10 \%$ FCS and $1 \%$ penicillin/streptomycin under standard culture conditions.

To generate cells that could inducibly express ATF $6^{1-373}$ and XBP1(s), we used the pRetro-tight system (Clontech). We transduced cells with viral particles containing pLenti-CMV-rtTA3 (Addgene plasmid \# 26429) and cultured cells in medium containing $5 \mu \mathrm{g} / \mathrm{L}$ blasticidin. Subsequently cells were transduced retrovirally with XBP1(s) or ATF $6^{1-373}$ constructs and cultured in medium containing $10 \mu \mathrm{g} / \mathrm{ml}$ puromycin. Stable transduction of cells containing the lentiviral pLKO_shPERK was achieved by transducing lentiviral particles into cells according to standard protocols. To generate cells that harbored stable expression of the constitutively active hamster Gadd34 ( ${ }^{\mathrm{CA}} \mathrm{Gad} 34$ ), the pCDNA3.1- ${ }^{\mathrm{CA}}$ Gadd34 construct was transfected in indicated cells using polyethyleneimine. Approximately 10E4 cells were seeded in $145 \mathrm{~cm}^{2}$ culture dishes and cultured with growth medium supplemented with $600 \mu \mathrm{g} / \mathrm{ml}$ G418 $\quad\left(\right.$ Gibco $^{\mathrm{mm}}$ LS10131027). After $\sim 2$ weeks, colonies were picked and clonally expanded for further experiments. All experiments were performed after $18 \mathrm{~h}$ of induction with doxycycline $(1 \mu \mathrm{g} / \mathrm{ml})$ unless specifically otherwise stated.

\section{Crystal violet}

For crystal violet analyses, cells were fixed with $4 \%$ paraformaldehyde in PBS for 15 min and subsequently immersed in $5 \mathrm{mg} / \mathrm{ml}$ crystal violet (Sigma C3886) in 2\% ethanol in $\mathrm{H}_{2} \mathrm{O}$. 


\section{EdU incorporation}

For measurement of EdU incorporation in cells, we used Click-iT $^{\circledR}$ EdU Alexa Fluor ${ }^{\circledR} 647$ (Thermofisher C10634) according to the manufacturer's protocol. The experiments were performed on the LSR Fortessa flow cytometer. Results were analyzed with FlowJo V10 software.

\section{ER tracker}

For analyses of the ER size, cells were plated on round $18 \mathrm{~mm}$ coverslips in a 12-well plate and stimulated with doxycycline $1 \mu \mathrm{g} / \mathrm{ml}$ after adhering. Cells were stained with ER-Tracker ${ }^{\mathrm{rm}}$ Red (BODIPY ${ }^{\mathrm{m}}$ TR Glibenclamide, Thermofisher \#E34250) and briefly fixated with $4 \%$ paraformaldehyde in PBS. Cells were imaged using confocal microscopy and ER surface was quantified using Image J software.

\section{Immunoblot}

For western blot analysis, cells were lysed in lysisbuffer (Cell Signaling) containing Protease Inhibitor Cocktail (Roche \#13538100), and sonicated. Samples were run on sodium dodecyl sulfate polyacrylamide gel electrophoresis gels under reducing conditions and transferred to a PVDF membrane. Specific detection was done by incubating the blot overnight in TBS with $0.1 \%$ Tween 20 with $1 \%$ BSA. Antibodies used for detection were anti-PERK (Cell Signaling \#3192, 1:1000), anti-BiP (Cell Signaling \#3183, 1:1000), anti-phospho-eIF2 $\alpha$ (Cell Signaling \#3398, 1:1000), anti-CHOP (Cell Signaling \#2895, 1:1000), antiXBP1 (Santa Cruz 7160, 1:500), Anti-eIF2 $\alpha$ (Cell Signaling \#2103, 1:1000), anti-c-Myc (Santa Cruz \#764, 1:1000), anti-ATF6 (Bioadacemia 73-500, 1:1000), anti-IRE1 $\alpha$ (Cell Signaling \#3294, 1:1000), and anti-beta actin (Sigma, A1978, 1:100,000). Secondary antibody detection with HRP labeled polyclonal antibodies was performed (Dako, Goat Anti-Rabbit \#P0448, Goat Anti-Mouse \#P0447, 1:2000), and antibody visualization was with Lumilight Plus (Roche,12015196001).

\section{S-methionine incorporation assay}

To measure global protein synthesis rates, we quantified the incorporation of ${ }^{35} \mathrm{~S}$-labeled methionine and cysteine into newly translated proteins. Cells were exposed to $15 \mathrm{~min}$ methionine starvation followed by a $45 \mathrm{~min}$ methionine incubation to label newly synthesized proteins, using $1 \mu \mathrm{l}(1.25 \mu \mathrm{Ci} / \mathrm{ml})$ of EasyTag ${ }^{\text {ma }} \mathrm{L}$-[35 S]Methionine (PerkerElmer ${ }^{\circledast}$ ) per well at $37 \mathrm{C}$, 95\% humidity and $5 \% \mathrm{CO}_{2}$ conditions. After labeling, cells were washed twice in cold PBS, harvested and centrifuged in cold PBS to remove supernatant. Next, cell pellets were lysed in cell lysis buffer (Cell Signaling). Lysate was blotted on labeled $24 \mathrm{~mm}$ glass microfiber filters (GF/C Whatman $^{\circledR}$ ) that were presoaked in $20 \%$ TCA. Filters were placed in a vacuum manifold and incubated in $10 \%$ ice-cold TCA for $15 \mathrm{~min}$, followed by $10 \%$ TCA at $90-95{ }^{\circ} \mathrm{C}$ for $10 \mathrm{~min}$. Filters were washed twice with cold $2 \%$ TCA and then twice with 95\% ethanol to remove TCA. Next, filters were air dried for $1 \mathrm{~h}$ at room temperature and placed in liquid scintillation cocktail (Ultima Gold, PerkerElmer ${ }^{\circledR}$. Radioactivity was quantified using a scintillation counter (Tri-Carb 2900TR).

\section{RNA extraction and quantitative RT-PCR}

For RNA purification, cells were lysed after $18 \mathrm{~h}$ of treatment with doxycycline $(1 \mu \mathrm{g} / \mathrm{ml})$ in $1 \mathrm{ml} \mathrm{TRI}$ Reagent $^{\circledR}$ (Sigma T9424). Further RNA purification was according to manufacturers' instructions. For cDNA synthesis, $1 \mu \mathrm{g}$ of RNA was transcribed using RevertAid reverse transcriptase (Thermo Scientific) using DNA hexamers of randomized sequence (Promega). Quantitative RT-PCR was performed using SensiFAST ${ }^{\mathrm{mm}}$ polymerase mix (Bioline 98020) according to manufacturers' protocol on a BioRad CFX96 Touch $^{\text {tw }}$ Real-Time PCR detection system using specific primers for the $m R N A$ of interest. RT-PCR primers (all human) were GAPDH forward 5'-AAGGTGAAGGTCGGAGTCAA-3' reverse $5^{\prime}$-AATGAAGGGGTCATTGATGG-3' ${ }^{\prime}$ XBP1(s) forward 5' - CCGCAGCAGGTGCAGG-3' reverse 5'-GAGTCAA TACCGCCAGAATCCA-3', ATF6 ${ }^{1-373}$ forward 5'- GC CTTTATTGCTTCCAGCAG-3' reverse 5'-TGAGACA GCAAAACCGTCTG-3', LUCIFERASE (firefly) forward 5'-TTACACCCGAGGGGGATGAT-3' reverse 5'-CCAG ATCCACAACCTTCGCT-3', GRP78 forward 5'-CATC ACGCCGTCCTATGTCG-3' reverse 5' -CGTCAAAGA CCGTGTTCTCG-3', HSP90B1 (GRP94) forward 5'-TG TAATTGCTGACCCAAGAGG-3' reverse $5^{\prime}$-TCCAA TTCAAGGTAATCAGATGC- $3^{\prime}$, DDIT3(CHOP) forward 5'-AGCCAAAATCAGAGCTGGAA-3' reverse 5'-TGG ATCAGTCTGGAAAAGCA-3', PERK forward 5'-TCA TCCAGCCTTAGCAAACC-3' reverse 5'-ATGCTTTC ACGGTCTTGGTC-3', DNAJB9 (ERDJ4) forward 5'- C AGCTCTTGTGGAGGAGCAG-3', ATF4 forward 5' -CA GCAGCACCAGGCTCT-3' reverse 5'-TCGAAGGTGT CTTTGTCGGT-3', EDEM1 forward 5'- GCTCAACCC CATCCACTG

-3' reverse 5'-CCAATGCATCAACAAGAGTCA-3', DNAJC3 (P58IPK) forward 5' - ACAAGGAAAACTTGA TGAAGCAG-3' reverse 5'-TGAGACTGTGCTTCCTT TTCTTC-3', DNAJB11 (HEDJ) forward 5'-GGATCTGG GTGCTGCTTATG-3' reverse 5'-TGTCTCCATGGGAG CTCTG-3', ASCL2 forward 5'-GGCACCAACACTTG GAGATT-3' reverse 5'-CCCTCCAGCAGCTCAAGT TA-3', AXIN2 forward 5'-TCTGGTGCAAAGACAT AGCCA- $3^{\prime}$ reverse 5'-AGTGTGAGGTCCACGAA AC-3', reverse $5^{\prime}-$ AATGCAGATTGCAAAGATGA AA-3', LGR5 forward 5'-GTTTCCCGCAAGA CGTAACT-3' reverse 5'- CAGCGTCTTCACCTCCT ACC-3', OLFM4 forward 5'-GTGGACAGAGT 
GGAACGCTT-3' reverse 5' - CACACTAATTAATTGG ACATATTCCCT-3', ha GADD34 forward 5'-CC ACCTGGAAGAGAGAGTGC-3' reverse $5^{\prime}$-GGGATCA GCTGAGAAAGACG-3', BMI1 forward 5'-CGT GTATTGTTCGTTACCTGGA-3' reverse 5'-TTCAGTA GTGGTCTGGTCTTGT-3', LRIG forward 5'-CTG GACGCGGAGCCTAAAC-3' reverse 5' ${ }^{\prime}$-TGTAG GTTCGGCAAGTCCTCA-3', HOPX 5'-GACAAGCA CCCGGATTCCA-3' reverse 5'-GTCTGTGACGGAT $^{\prime}$ CTGCACTC-3', TERT forward 5'-TCACGGAGAC CACGTTTCAAA-3' reverse $5^{\prime}$-TTCAAGTGCTGTCTG ATTCCAAT-3'.

\section{Statistics}

Statistical analyses were performed using GraphPad Prism software (Graphpad, La Jolla, CA). All data are presented as mean \pm SEM of three independent experiments of technical triplicates. For comparison of two groups, Student's $t$-test was used, for grouped analyses, two-way ANOVA was used followed by Bonferroni's posthoc test for multiple comparisons. $P$ value of $<0.05$ was deemed significant.

\section{Results}

Activation of XBP1(s) and ATF6 results in upregulation of UPR target genes

We set out to examine effects of UPR transcription factors XBP1 and ATF6 on intestinal epithelium. To this end, we utilized LS174T colorectal cancer cells that harbor mutations in the WNT signaling pathway, causing them to resemble cells of the intestinal crypt. These cells have the possibility to differentiate and express markers of terminally differentiated cells of the intestine, such as mucins and cyclin dependent kinase inhibitor P21 ${ }^{31,32}$. We transduced LS174T cells with constructs enabling doxycycline inducible expression of transcription factors XBP1 and ATF6. Under homeostatic conditions, levels of ER stress are low and transcripts of XBP1 and ATF6 encode for proteins that are not transcriptionally active. We therefore generated cells that carried tetracycline inducible transcripts of active XBP1 (XBP1(s)) and of the transcriptionally active cytosolic domain (amino acids 1 to 373) of ATF6 (further referred to as ATF6 $\left.{ }^{1-373}\right)^{26}$.

Using quantitative PCR, we confirmed expression of $\mathrm{XBP} 1(\mathrm{~s})$ and found it harbored transcriptional activity, as judged by increased expression of direct target genes $P 58^{I P K}$, ERDJ4, ER chaperones GRP78, GRP94 and UPR transcription factor $C H O P$ (Fig. 1a) ${ }^{29}$. Among upregulated genes, we found that UPR effector PERK was upregulated 2.8-fold, confirming previous reports that identify PERK as a direct transcriptional target of $\mathrm{XBP} 1(\mathrm{~s})^{23}$.

Immunoblots of XBP1(s) cells confirmed high expression of XBP1(s), which reproducibly had a slightly lower molecular weight than endogenous XBP1(s). XBP1(s)- expressing cells exhibited upregulation of target chaperone GRP78. Expression of XBP1(s) and GRP78 were upregulated to a similar extent as we observed in control cells after treatment with thapsigargin, a chemical inducer of ER-stress (Fig. 1b and Supp. Fig. 1A). It has been reported that IRE1 $\alpha$ protein levels increase upon deletion of $\mathrm{XBP} 1^{25}$. We found no altered expression in IRE1 $\alpha$ protein level upon enforced expression of XBP1(s), while thapsigargin treatment increased the protein levels as expected. We additionally confirmed that expression of transcription factor XBP1(s) was indeed localized in the nucleus (Fig. 1c). It has been established that XBP1(s) signaling increases the capacity of the ER by inducing ERbiogenesis ${ }^{20}$. We therefore next analyzed the volume of the ER upon induction of XBP1(s). Indeed, we found that enforced expression of XBP1(s) resulted in increased size of the ER to a similar extent as we observed after treatment with thapsigargin (Supp Fig. 1B).

Parallel to XBP1(s) expression, ATF6 expression is linked to ER expansion and upregulation of ER chaperones $^{26,28}$. Similar to our observations in XBP1(s)-expressing cells, we confirmed upregulation of ATF6 $6^{1-373}$ protein upon induction with doxycycline in ATF $6^{1-373}$ cells. Upregulation of ATF6 ${ }^{1-373}$ and GRP78 was to a similar extent as in control cells treated with thapsigargin (Supp. Fig. 1C, D, E). Furthermore, IRE1 $\alpha$ levels were not grossly altered upon ATF $6^{1-373}$ expression. In addition, we confirmed transcriptional activity of $\mathrm{ATF}^{1-373}$ in stably transfected LS174T cells. Moreover, we observed expected upregulation of UPR target genes on $m R N A$ and protein level upon ATF6 ${ }^{1-373}$ overexpression (Fig. 1d, e).

ATF6 ${ }^{1-373}$-expressing cells had a similar increase in ER size (Supp. Fig. 1F). Although PERK $m R N A$ is increased by direct binding of XBP1 to the PERK promoter, this has not been demonstrated for ATF6. We did, however, observe that expression of ATF6 ${ }^{1-373}$ resulted in 13-fold upregulation of PERK $m R N A$.

These results verify that overexpression of XBP1(s) or ATF $6^{1-373}$ results in upregulation of UPR target genes and increased size of the ER.

\section{Expression of $\mathrm{XBP} 1(\mathrm{~s})$ and $\mathrm{ATF} 6^{1-373}$ results in reduced stemness and proliferation}

We have previously shown that general UPR activation results in PERK-dependent reduction of intestinal epithelial stemness ${ }^{7}$. Moreover, mice that lack UPR transcription factor $X b p 1$ in the intestinal epithelium display increased intestinal stem cell numbers ${ }^{25}$. We next examined stemness in cells that had enforced expression of XBP1(s). To this end we performed quantitative RT-PCR for intestinal stem cell marker genes LGR5 and OLFM4 and found that these were significantly decreased upon induction of XBP1(s) (Fig. 2a). Stem cell genes LGR5 and OLFM4 mark crypt base columnar stem cells that harbor 


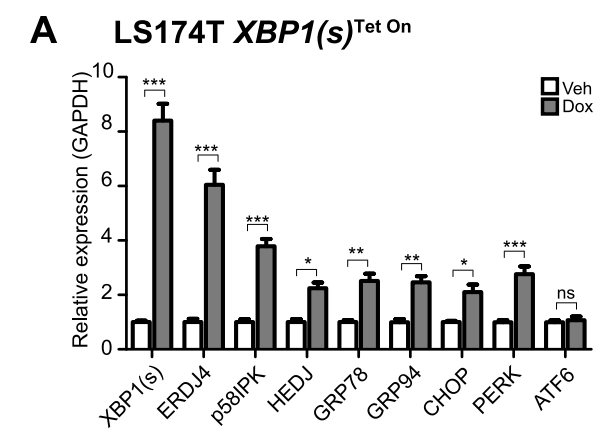

D LS174T ATF6 ${ }^{1-373}$ Tet on

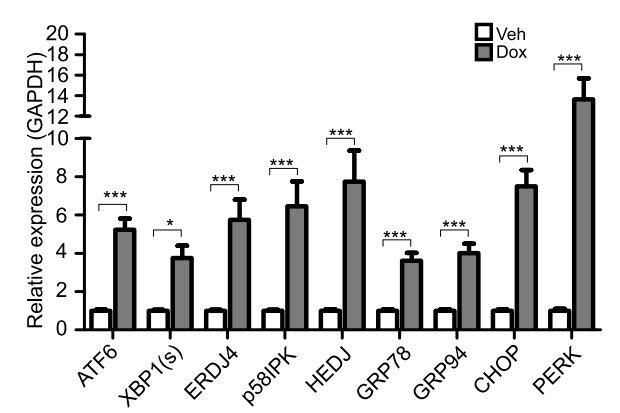

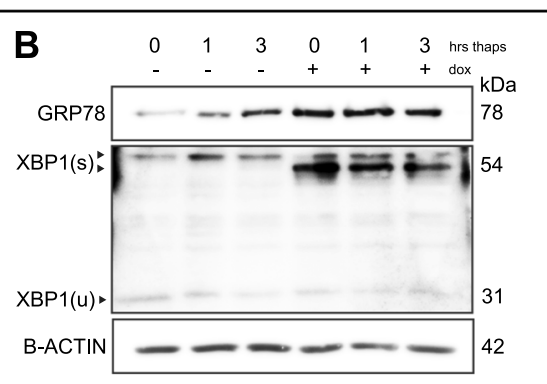

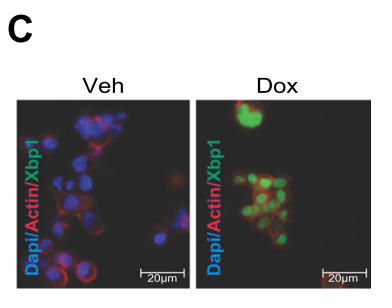

E

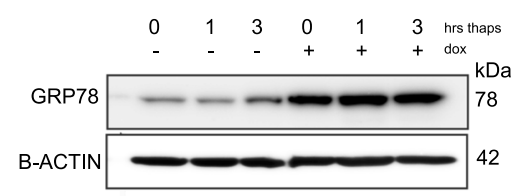

Fig. 1 LS174T cells expressing XBP1(s) or ATF6 ${ }^{1-373}$ upregulate general UPR target genes. a Quantitative RT-PCR analysis for XBP1(s) and downstream target genes upon induction of XBP1(s) expression. b Protein level of XBP1(s) and GRP78 in LS174T XBP1(s) Tet On cells. Cells were incubated for 0,1 or $3 \mathrm{~h}$ with thapsigargin $200 \mathrm{nM}$. Note that transgenic XBP1(s) had a slightly lower molecular weight than endogenous XBP1(s). $c$ Immunostaining of XBP1 in LS174T XBP1(S) ${ }^{\text {Tet On }}$ cells. $\mathbf{d}$ Quantitative RT-PCR analysis for ATF6 ${ }^{1-373}$ and downstream target genes upon induction of ATF6 ${ }^{1-373}$ expression. e Protein level of GRP78 in LS174T ATF6 ${ }^{1-373 ~ T e t ~ O n ~ c e l l s . ~ C e l l s ~ w e r e ~ t r e a t e d ~ w i t h ~ v e h i c l e ~ o r ~ d o x y c y c l i n e ~} 1 \mu \mathrm{gg} / \mathrm{mL}$ for $18 \mathrm{~h}$. All data are shown as means \pm SEM. ${ }^{*} P<0.05,{ }^{*} P<0.01,{ }^{* *} P<0.001$

high WNT signaling. In past years, a number of stem cell markers have been described that mark alternatively localized stem cells, which are not driven by WNT signaling, including BMI1, LRIG, HOPX and TERT ${ }^{33-36}$. We examined $m R N A$ expression of these stem cell markers, and found only TERT to be reduced upon induction of both XBP1(s) and ATF6 $6^{1-373}$. Other markers were unaltered of even slightly elevated (Supp. Fig. 2A and B). These results show that in LS174T cells, which are cells with high WNT signaling, stem cell markers that are driven by WNT signaling are reduced upon expression of $\mathrm{XBP} 1(\mathrm{~s})$ and ATF6 ${ }^{1-373}$, whereas WNT independent alternative stem cell markers are largely unaffected.

Additionally, XBP1(s) expression resulted in significant decrease in cell numbers (Fig. 2b). This was, at least in part, accounted for by a decrease in cellular proliferation of $60 \%$ as measured by EdU incorporation (Fig. 2c). Similar to XBP1(s)-expressing cells, ATF $6^{1-373}$ expression resulted in decreased cellular proliferation and decreased expression of stem cell markers (Fig. 2d, e). Moreover upon ATF6 ${ }^{1-373}$ expression, EdU incorporation was decreased (Fig. 2f).

We have previously shown that induction of UPR activity may result in loss of stemness through rapid loss of short lived transcription factors that are critical for the stem cell phenotype (such as C-MYC) we tested whether expression of C-MYC was altered upon XBP1(s) induction $^{7,37}$. Indeed, we found markedly reduced expression of C-MYC protein after induction of XBP1(s) (Supp. Fig. 2C).

To exclude a cell line specific effect of the observed growth disadvantage upon expression of XBP1(s) or ATF $6^{1-373}$, we generated two alternative colorectal cancer cell lines harboring inducible expression of XBP1(s) and ATF6 $^{1-373}$ (SW480 and DLD-1, Supp. Fig. 3A). Similar to what we observed in LS174T cells, Expression of either $\mathrm{XBP} 1(\mathrm{~s})$ or $\mathrm{ATF} 6^{1-373}$ resulted in reduced cell viability (Supp. Fig. 3B). We could however not detect any $m R N A$ of OLFM4 in both these cell lines, and SW480 cells did not express mRNA of LGR5 and therefore continued experiments with LS174T cells solely.

To rule out ER stress and subsequent UPR activation through high upregulation of transgenic proteins or through heterotopic actions of doxycycline, we generated control cells expressing high levels of firefly luciferase in a doxycycline responsive manner (LS174T Luciferase ${ }^{\mathrm{TetOn}}$ ). In these cells we observed no effects on expression of UPR target genes or stem cell markers as well as unaltered growth (Supp. Fig. 3C-H). 
A

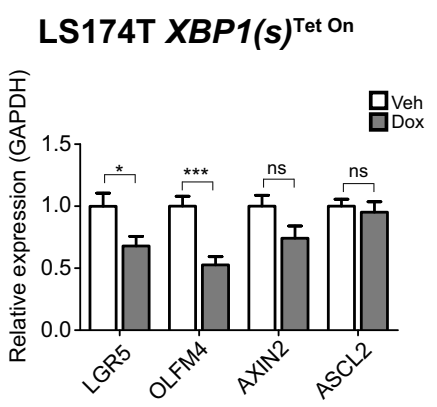

D

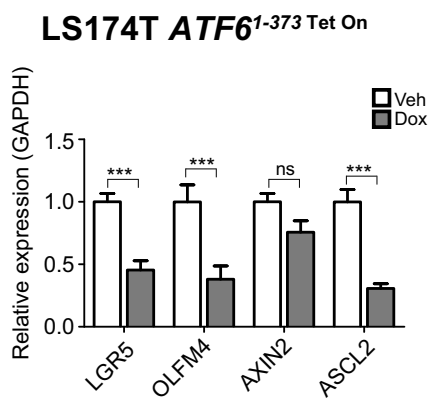

B

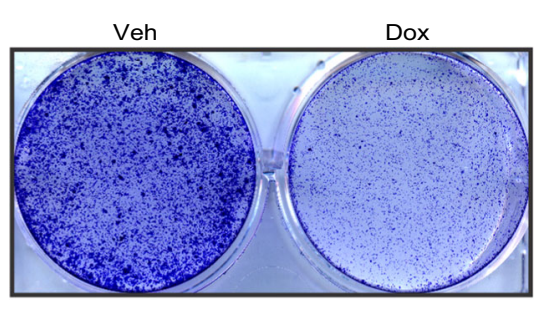

$\mathbf{E}$

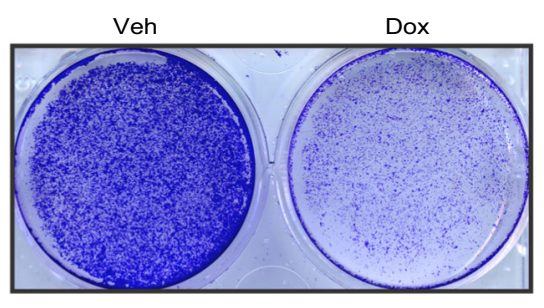

C

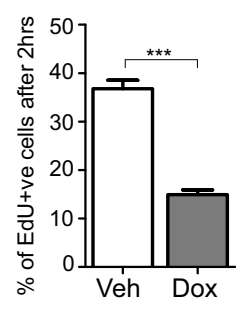

$\mathbf{F}$

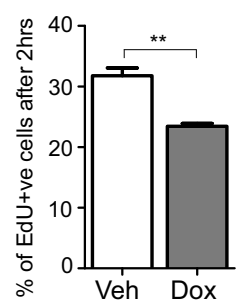

Fig. 2 XBP1(s) or ATF6 ${ }^{1-373}$ expression results in reduced stemness and cell proliferation in LS174T colorectal cancer cells. a Quantitative RT-PCR analysis for intestinal stem cell markers LGR5, OLFM4 and ASCL2 or Wht target genes LGR5 and AXIN2 in LS174T XBP1(S) ${ }^{\text {Tet On }}$ cells. b Crystal violet cell viability assay in $L S 174 T$ XBP1(s) $)^{\text {Tet On }}$ cells. c FACS-based EdU incorporation assay in LS174T XBP1(s) $)^{\text {Tet On }}$ cells; the assay was performed after $2 \mathrm{~h}$ of EdU incorporation. d Quantitative RT-PCR analysis for intestinal stem cell markers LGR5, OLFM4 and ASCL2 or Wnt target genes LGR5 and AXIN2 in LS174T ATF6 ${ }^{1-373}$ Tet On cells. e Crystal violet cell viability assay in LS174T ATF6 ${ }^{1-373}$ Tet On cells. f FACS-based EdU incorporation assay in LS174T ATF6 ${ }^{1-373}$ Tet On cells; the assay was performed after $2 \mathrm{~h}$ of EdU incorporation. All data are shown as means $\pm \mathrm{SEM}$. ${ }^{*} P<0.05$, ${ }^{* *} P<0.01,{ }^{* * *} P<0.001$

Thus, enforced expression of XBP1(s) and ATF $6^{1-373}$ in colon cancer cells results in decrease in stemness and proliferation, not attributable to doxycycline.

\section{Activation of XBP1(s) and ATF6 ${ }^{1-373}$ enhances sensitivity to UPR activation}

Enforced expression of XBP1(s) or ATF6 $6^{1-373}$, even in the absence of ER stress, increased the size of the ER and the expression of ER chaperones. ER chaperones such as GRP78 are needed for proper folding of unfolded proteins to restore ER homeostasis. We therefore hypothesized that a higher amount of chaperones and an increased ER size, resulting from transgenic expression of XBP1(s) or ATF $6^{1-373}$, could result in increased resistance to ER stress. We treated cells with a very low level of thapsigargin intended to yield no growth impairment in control cells. To our surprise, cells with enforced expression of XBP1(s) or ATF $6^{1-373}$ had reduced cell viability after a short pulse exposure to these low levels of thapsigargin (Supp. Fig. 4A, B). These results indicate that enforced expression of XBP1 (s) or ATF $6^{1-373}$ reduces the threshold for activation of the UPR and enhances sensitivity to ER stress.
Expression of XBP1(s) and ATF6 ${ }^{1-373}$ causes activation of PERK and inhibition of global protein synthesis

Our observations of growth reduction and increased expression of PERK mRNA in XBP1(s) and ATF6 ${ }^{1-373}$ expressing cell lines may result from downstream activation of PERK. We therefore analyzed activity of PERK upon induction of XBP1(s) or ATF6 ${ }^{1-373}$. Indeed, PERK protein was upregulated and downstream phosphorylation of eIF2 $\alpha$ was observed (Fig. 3a and B). Phosphorylation of eIF2 $\alpha$ results in a transient inhibition of global protein translation ${ }^{13}$. We therefore analyzed cellular global protein synthesis using radioactively labeled 35Smethionine. Expression of XBP1(s) and ATF $6^{1-373}$ resulted in a significant decrease in protein synthesis (Fig. 3c, d) ${ }^{38}$. To study the kinetics by which induction of XBP1(s) and ATF6 resulted in eIF2 $\alpha$ phosphorylation and loss of markers of stemness, we treated cells for different durations with doxycycline. Interestingly, we found that phosphorylation of eIF $2 \alpha$ occurred slightly earlier upon ATF6 induction than upon XBP1(s) induction (Supp. Fig. $5 \mathrm{~A}, \mathrm{~B})$. In addition, decrease of LGR5 $m R N A$ was incremental over time in both cell lines (Supp. Fig. 5C, D). 


\section{A LS174T XBP1(s) $)^{\text {Tet On }}$}

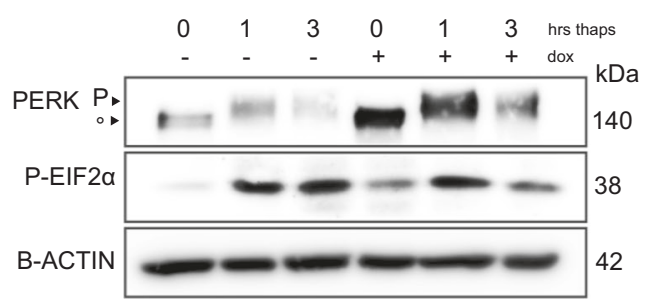

C

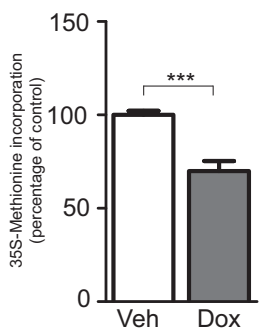

D

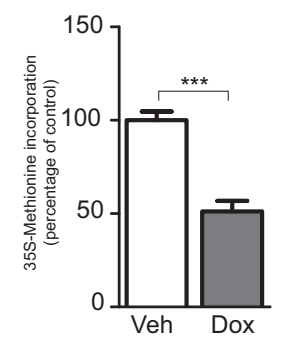

B LS174T ATF6 ${ }^{1-373 \text { Tet On }}$

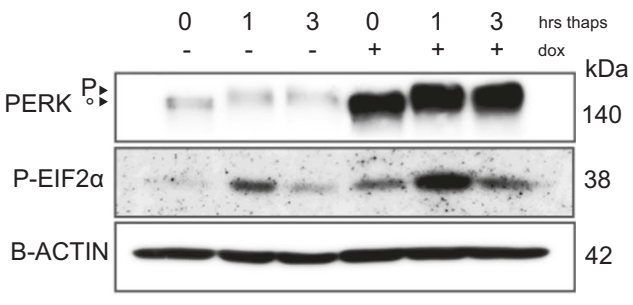

$\mathbf{E}$

$\mathbf{F}$

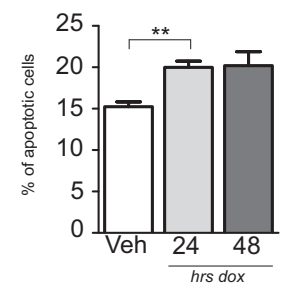

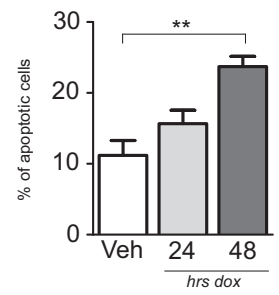

Fig. 3 XBP1(s) or ATF6 ${ }^{1-373}$ expression upregulates PERK-elF2a and inhibits global translation. $\mathbf{a}, \mathbf{c}$, e $L S 174 T X B P 1(s)^{T e t}$ On $c e l l s . \mathbf{b}, \mathbf{d}, \mathbf{f} L S 174 T$

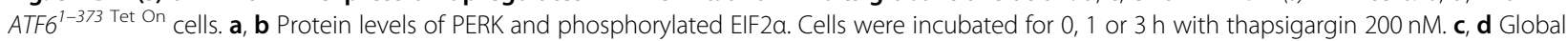
protein translation rate, measured by ${ }^{355}$ Methionine incorporation assay. e, $\mathbf{f}$ Percentage of apoptotic cells, measured with propidium iodide and Annexin $\vee$ staining on Flow Cytometry. All data are shown as means \pm SEM. ${ }^{*} P<0.05,{ }^{* *} P<0.01,{ }^{* *} P<0.001$

Previous reports have shown that high and prolonged CHOP signaling, downstream of phosphorylated eIF2 $\alpha$, leads to apoptosis ${ }^{39-41}$. We therefore determined whether prolonged XBP1(s) or ATF6 ${ }^{1-373}$ signaling, with established activation of PERK- phospho-eIF2 $\alpha$, led to an increase in apoptotic cells. We measured both early and late apoptosis with Propidium Iodide (PI) and Annexin V staining on flow cytometry. The number of apoptotic cells was increased by $31 \%$ after induction of XBP1(s) (Fig. 3e). In the ATF6 ${ }^{1-373}$-expressing cells, apoptosis was increased with $39 \%$ (Fig. 3f).

We conclude that activation of XBP1(s) and ATF6 ${ }^{1-373}$ is capable of activating PERK-eIF2 $\alpha$ signaling, which might account for reduced global protein synthesis rates and induced apoptosis in these cells.

\section{XBP1(s) and ATF $6^{1-373}$ activation results in cell cycle arrest in distinct phases}

It has been described that UPR activation through PERK signaling can result in cell cycle arrest in both G1 and G2 phases. G1 arrest is thought to be mediated by Cyclin D1 abrogation, whereas G2 arrest results from Chk1 effector kinase activation $^{15,42,43}$. To further elucidate how XBP1(s) and ATF $6^{1-373}$ expression resulted in growth arrest, we performed cell cycle analysis using Propidium Iodide. Similar to thapsigargin treatment, XBP1(s) expression arrested cells in the G1 phase (Supp. Fig. 6A) ${ }^{15}$. Interestingly, ATF $6^{1-373}$-expressing cell lines upregulated
PERK-eIF2 $\alpha$ to the same extent, though ATF6 ${ }^{1-373}$ expression arrested cells in the G2 phase (Supp Fig. 6B).

\section{XBP1(s)-induced growth arrest is mediated by PERK-elF2 $a$ signaling}

Previously, we have shown that PERK-eIF2 $\alpha$ signaling is important for the decreased stemness that is observed in colorectal cancer cells after induction of ER stress. Moreover, reversing eIF2 $\alpha$ phosphorylation by continuous dephosphorylation of eIF $2 \alpha$ using GADD34 was capable of rescuing loss of markers of stemness in these cells $^{7,30}$. To further implicate PERK-eIF2 $\alpha$ signaling in $\mathrm{XBP} 1$ (s) and ATF6 ${ }^{1-373}$ induced growth retardation, we transduced cells with lentiviral particles containing short hairpin RNA fragments (shRNA) directed against PERK.

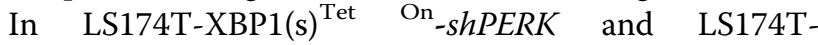
ATF6 ${ }^{1-373 T e t}$ On - shPERK cells, PERK expression was significantly reduced on both $m R N A$ and protein levels (Supp Fig. 7A-D). Downstream phosphorylation of eIF $2 \alpha$ was however only mildly reduced and downstream activation of eIF $2 \alpha$ targets ATF4 and CHOP were not inhibited. Reduced cell viability upon XBP1(s) or ATF6 ${ }^{1-373}$ expression was unaltered in cells expressing PERK shRNA (Supp Fig. 7E, F). Lack of inhibition of PERK targets may be accounted for by activation of redundant eIF2 $\alpha$-kinase upon induction of ER-stress ${ }^{38}$. To overcome redundancy, we generated XBP1(s) and ATF $6^{1-373}$-expressing cells that stably expressed high levels of a constitutively active 


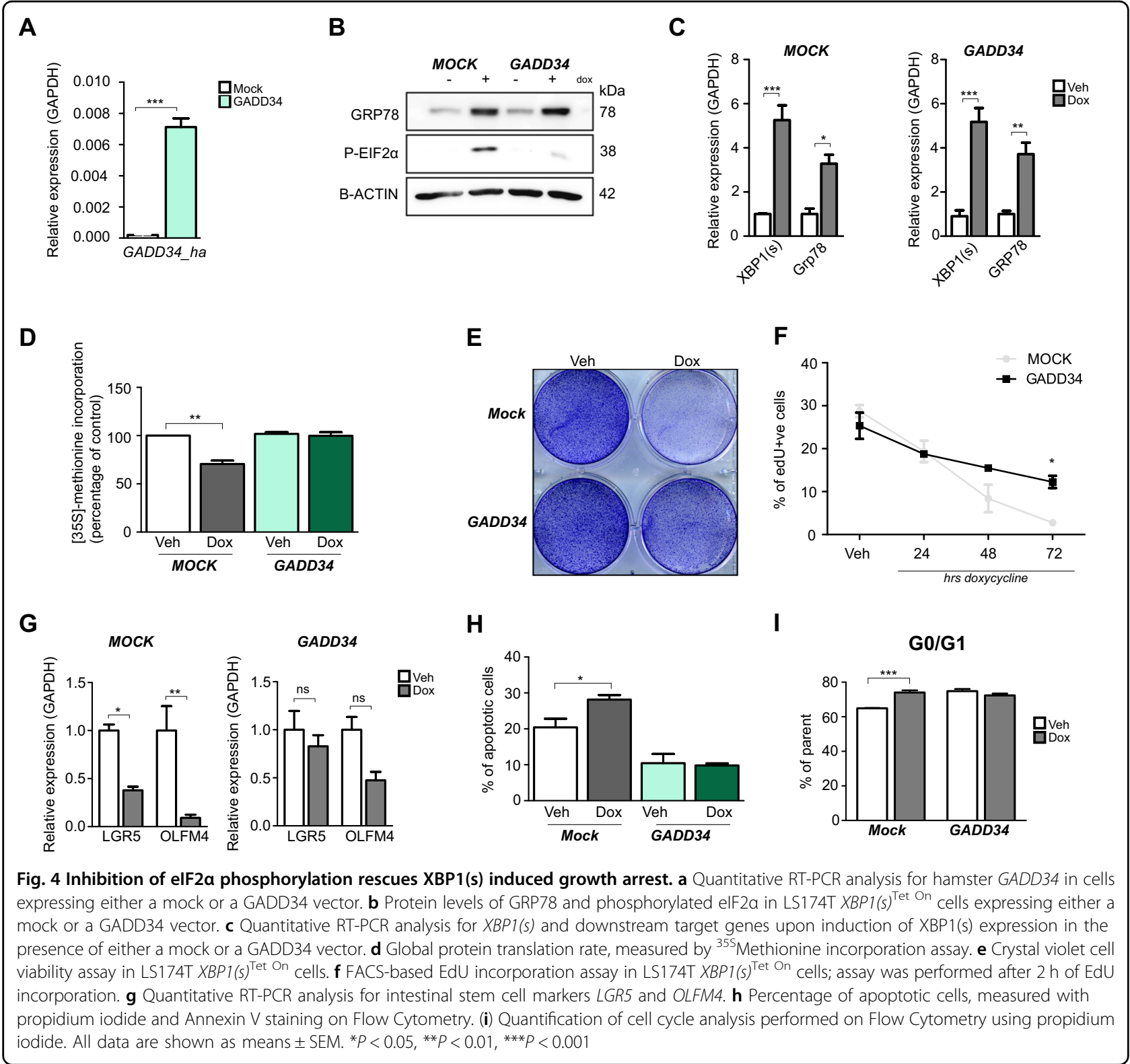

form of GADD34 (Fig. 4a). Interestingly, in LS174TATF $6^{1-373}$ cells, transgenic GADD34 expression abated over a short period of time in multiple distinct clonally grown cell lines and eIF $2 \alpha$ phosphorylation was not satisfactorily inhibited. We could however generate LS174T-XBP1(s) ${ }^{\text {Tet On }}$-GADD34 cells in which only very modest levels of eIF $2 \alpha$ phosphorylation could be observed upon induction of XBP1(s) (Fig. 4b), though increase in $X B P 1(s) m R N A$ and its downstream UPR target genes was similar to observed in the parental XBP1(s)-expressing cell line (Fig. 4c). In addition, GADD34 expression did not alter GRP78 protein levels. We found that in LS174TXBP1(s) ${ }^{\text {Tet }}$ On - GADD34 cells, reduced global protein synthesis, as observed in XBP1(s) cells was fully rescued (Fig. 4d). In addition, in LS174T-XBP1(s) ${ }^{\text {Tet On }-G A D D 34, ~}$ reduced proliferation was largely rescued (Fig. 4e, f). Moreover, stem cell markers were largely normalized upon induction of XBP1(s) in these cells (Fig. 4g). Incomplete normalization of stem cell markers may have resulted from remaining modest levels of phosphorylated eIF $2 \alpha$. Potentially, rescue of XBP1(s) induced growth arrest in GADD34-expressing cells resulted from decreased apoptosis (Fig. 4h). Interestingly, cell cycle arrest in G0/1 upon doxycycline could be rescued in the GADD34-expressing cells, though already a larger percentage of unstimulated cells expressing GADD34, were 
in the G0/1 phase of the cell cycle (Fig. 4i). These results suggest that XBP1(s)-induced effects on cellular proliferation, translation and apoptosis are mediated by PERK-eIF2 $\alpha$ signaling.

\section{Discussion}

The UPR is a signaling pathway that controls cellular homeostasis at the level of protein quality. Accumulating evidence points towards a role of the UPR in cell fate decisions such as differentiation of intestinal epithelial stem cells and colorectal cancer cells. We investigated differential roles for distinct UPR components in these processes.

It has previously been shown that activation of PERKeIF2 $\alpha$ signaling results in reduced proliferation and increased apoptosis ${ }^{44}$. Moreover, we have previously implicated this pathway in loss of intestinal epithelial stemness and enforced differentiation ${ }^{7}$. Our current experiments show that activation of the two alternative branches of the UPR, XBP1 and ATF6, also results in reduced proliferation and diminished stemness. We have corroborated reduced cell viability experiments in three cell lines, leading us to think that this phenomenon is not cell-line specific. These results are in line with studies showing that epithelial loss of Xbp1 in mice resulted in increased stem cell numbers and increased tumorigenesis ${ }^{25}$. Moreover, increased apoptosis upon ATF6 expression in chondrocytes and myoblasts corroborates these findings ${ }^{45,46}$. In contrast, two studies report pro-tumorigenic properties of cells upon overexpression of XBP1 or ATF $6^{47,48}$. These effects of XBP1 and ATF6 may result from expression in alternative cell types or stable expression of XBP1 and ATF6 instead of inducible expression, which may have resulted in selection of cells.

Our findings that XBP1 and ATF6 expression result in reduced proliferation and stemness are further strengthened by the fact that these cells exhibit reduced global protein synthesis. In a previous report, it was shown that XBP1(s) and ATF6 ${ }^{1-373}$ expression in HEK293T cells did not result in reduced protein production capacity. In these reports, a small but non-significant reduction in $35 \mathrm{~S}$ incorporation was noted in the XBP1(s) expressing HEK293T cells after only a short induction period of $12 \mathrm{~h}$. Discrepancy with significant reduction in $35 \mathrm{~S}$ incorporation in our experiments may depend on this short induction period, potentially preceding significant reduction of translation ${ }^{49}$.

LS174T cells that express XBP1 or ATF6 exhibit increased sensitivity to thapsigargin. Interestingly, in most models examining either gain or lack of activity of the principal UPR components, sensitivity to ER stress increased. This was shown for PERK ${ }^{-1-}$ mouse embryonic stem cells, homozygous or heterozygous knockout of Xbp1 and loss of Atf6 ${ }^{50-52}$. ATF6 ${ }^{-1-}$ and IRE1 $\alpha^{-/-}$melanoma cells showed increased sensitivity to ER stress in the presence of adequate PERK signaling. Likely, balance in the UPR is regulated to such an extent that perturbation of any UPR component may result in increased sensitivity to ER stress, resulting in reduced viability.

Colorectal cancer cells that harbor XBP1 or ATF6 activation have a highly similar phenotype, potentially resulting from the combined transcriptional activity of these factors as heterodimers ${ }^{53}$. We find remarkable differences, however, in alteration of the cell cycle phase upon XBP1 or ATF6. Although it has been advocated that PERK activation can both lead to $\mathrm{G} 1$ and $\mathrm{G} 2$ arrest via different mechanisms, the differences between XBP1 induced G1 arrest and ATF6 induced G2 arrest remain to be elucidated ${ }^{15,42,43}$.

XBP1 and ATF6 both activate PERK-eIF2 $\alpha$ signaling. Interestingly, phenotypical changes upon XBP1 induction, such as reduced growth, cell cycle arrest, and reduced global protein synthesis, result from reduced eIF2 $\alpha$ signaling, since a rescue of this phenotype resulted from dephosphorylation of eIF2 $\alpha$.

Our experiments uncover a novel cross-interaction between activated XBP1 and ATF6 and PERK-eIF2 $\alpha$. We uncover that the interaction between XBP1 and PERKeIF $2 \alpha$ is mechanistically responsible for the antiproliferative phenotype of XBP1, which can be rescued. Our data show again the importance of PERK-eIF2 $\alpha$ in cellular viability and stemness. These results may be utilized to target PERKeIF $2 \alpha$ in treatment or prevention of intestinal malignancies.

\section{Acknowledgements}

This work was supported by a grant of the Dutch Cancer Foundation (KWF/ Alpe 11053/2017-1) and by a grant of the Netherlands Organisation for Scientific Research (NWO-Veni 91615032).

\section{Author details}

${ }^{1}$ Amsterdam UMC, University of Amsterdam, Department of Gastroenterology and Hepatology, Tytgat Institute for Liver and Intestinal Research, Meibergdreef 71, Amsterdam, The Netherlands. ${ }^{2}$ Amsterdam UMC, University of Amsterdam, Department of Internal Medicine and Hematology, Meibergdreef 9, Amsterdam, The Netherlands. ${ }^{3}$ Present address: Roche Innovation Center Basel, F. Hoffmann-La Roche AG, Basel, Switzerland

Conflict of interest

The authors declare that they have no conflict of interest.

\section{Publisher's note}

Springer Nature remains neutral with regard to jurisdictional claims in published maps and institutional affiliations.

Supplementary Information accompanies this paper at (https://doi.org/ 10.1038/s41419-019-1729-4)

Received: 14 January 2019 Revised: 18 May 2019 Accepted: 20 May 2019 Published online: 21 June 2019

\section{References}

1. Clevers, H. The intestinal crypt, a prototype stem cell compartment. Cell 154, 274-284 (2013).

2. Barker, N. et al. Crypt stem cells as the cells-of-origin of intestinal cancer. Nature 457, 608-611 (2009).

3. Fearon, E. R. \& Vogelstein, B. A genetic model for colorectal tumorigenesis. Cell 61, 759-767 (1990). 
4. Vogelstein, B. et al. Genetic alterations during colorectal-tumor development. N. Engl. J. Med. 319, 525-532 (1988).

5. Dylla, S. J. et al. Colorectal cancer stem cells are enriched in xenogeneic tumors following chemotherapy. PloS ONE 3, e2428 (2008).

6. Jin, X., Jin, X. \& Kim, H. Cancer stem cells and differentiation therapy. Tumour Biol.: J. Int. Soc. Oncodev. Biol. Med. 39, 1010428317729933 (2017).

7. Heijmans, J. et al. ER stress causes rapid loss of intestinal epithelial stemness through activation of the unfolded protein response. Cell Rep. 3, 1128-1139 (2013).

8. Ma, Y. \& Hendershot, L. M. The unfolding tale of the unfolded protein response. Cell 107, 827-830 (2001).

9. Harding, H. P., Calfon, M., Urano, F., Novoa, I. \& Ron, D. Transcriptional and translational control in the Mammalian unfolded protein response. Annu. Rev. Cell Dev. Biol. 18, 575-599 (2002).

10. Van Lidth de Jeude, J. F. et al. Induction of endoplasmic reticulum stress by deletion of Grp78 depletes Apc mutant intestinal epithelial stem cells. Oncogene 36, 3397-3405 (2017).

11. Van Lidth de Jeude, J. F. et al. Heterozygosity of chaperone Grp78 reduces intestinal stem cell regeneration potential and protects against adenoma formation. Cancer Res. (2018).

12. Hetz, C. The unfolded protein response: controlling cell fate decisions under ER stress and beyond. Nat. Rev. Mol. Cell Biol. 13, 89-102 (2012).

13. Harding, H. P., Zhang, Y. \& Ron, D. Protein translation and folding are coupled by an endoplasmic-reticulum-resident kinase. Nature 397, 271-274 (1999).

14. Kimball, S. R. Eukaryotic initiation factor elF2. Int. J. Biochem. Cell Biol. 31, 25-29 (1999).

15. Brewer, J. W. \& Diehl, J. A. PERK mediates cell-cycle exit during the mammalian unfolded protein response. Proc. Natl Acad. Sci. USA 97, 12625-12630 (2000).

16. Ramsay, G., Stanton, L., Schwab, M. \& Bishop, J. M. Human proto-oncogene Nmyc encodes nuclear proteins that bind DNA. Mol. Cell Biol. 6, 4450-4457 (1986).

17. Hann, S. R. \& Eisenman, R. N. Proteins encoded by the human c-myc oncogene: differential expression in neoplastic cells. Mol. Cell Biol. 4, 2486-2497 (1984).

18. Vattem, K. M. \& Wek, R. C. Reinitiation involving upstream ORFs regulates ATF4 mRNA translation in mammalian cells. Proc. Natl Acad. Sci. USA 101, 11269-11274 (2004).

19. Calfon, M. et al. IRE1 couples endoplasmic reticulum load to secretory capacity by processing the XBP-1 mRNA. Nature 415, 92-96 (2002).

20. Sriburi, R., Jackowski, S., Mori, K. \& Brewer, J. W. XBP1: a link between the unfolded protein response, lipid biosynthesis, and biogenesis of the endoplasmic reticulum. J. Cell Biol. 167, 35-41 (2004).

21. Back, S. H. et al. Translation attenuation through elF2alpha phosphorylation prevents oxidative stress and maintains the differentiated state in beta cells. Cell Metab. 10, 13-26 (2009).

22. Reimold, A. M. et al. Plasma cell differentiation requires the transcription factor XBP-1. Nature 412, 300-307 (2001).

23. Acosta-Alvear, D. et al. XBP1 controls diverse cell type- and condition-specific transcriptional regulatory networks. Mol. Cell 27, 53-66 (2007).

24. Shaffer, A. L. et al. XBP1, downstream of Blimp-1, expands the secretory apparatus and other organelles, and increases protein synthesis in plasma cell differentiation. Immunity 21, 81-93 (2004).

25. Niederreiter, L. et al. ER stress transcription factor Xbp1 suppresses intestinal tumorigenesis and directs intestinal stem cells. J. Exp. Med. 210, 2041-2056 (2013).

26. Haze, K., Yoshida, H., Yanagi, H., Yura, T. \& Mori, K. Mammalian transcription factor ATF6 is synthesized as a transmembrane protein and activated by proteolysis in response to endoplasmic reticulum stress. Mol. Biol. Cell 10, 3787-3799 (1999)

27. $\mathrm{Ye}_{\mathrm{e}} \mathrm{J}$. et al. ER stress induces cleavage of membrane-bound ATF6 by the same proteases that process SREBPs. Mol. Cell 6, 1355-1364 (2000).

28. Bommiasamy, $H$. et al. ATF6alpha induces XBP1-independent expansion of the endoplasmic reticulum. J. Cell Sci. 122, 1626-1636 (2009).
29. Lee, A. H., Iwakoshi, N. N. \& Glimcher, L. H. XBP-1 regulates a subset of endoplasmic reticulum resident chaperone genes in the unfolded protein response. Mol. Cell Biol. 23, 7448-7459 (2003).

30. Novoa, I., Zeng, H., Harding, H. P. \& Ron, D. Feedback inhibition of the unfolded protein response by GADD34-mediated dephosphorylation of elF2alpha. J. Cell Biol. 153, 1011-1022 (2001).

31. Tom, B. H. et al. Human colonic adenocarcinoma cells. I. Establishment and description of a new line. In Vitro 12, 180-191 (1976).

32. van de Wetering, $M$. et al. The beta-catenin/TCF-4 complex imposes a crypt progenitor phenotype on colorectal cancer cells. Cell 111, 241-250 (2002).

33. Sangiorgi, E. \& Capecchi, M. R. Bmi1 is expressed in vivo in intestinal stem cells. Nat. Genet. 40, 915-920 (2008).

34. Montgomery, R. K. et al. Mouse telomerase reverse transcriptase (mTert) expression marks slowly cycling intestinal stem cells. Proc. Natl Acad. Sci. USA 108, 179-184 (2011).

35. Takeda, N. et al. Interconversion between intestinal stem cell populations in distinct niches. Science 334, 1420-1424 (2011).

36. Powell, A. E. et al. The pan-ErbB negative regulator Lrig1 is an intestinal stem cell marker that functions as a tumor suppressor. Cell 149, 146-158 (2012).

37. Muncan, $\vee$. et al. Rapid loss of intestinal crypts upon conditional deletion of the Wnt/Tcf-4 target gene c-Myc. Mol. Cell Biol. 26, 8418-8426 (2006).

38. Hamanaka, R. B., Bennett, B. S., Cullinan, S. B. \& Diehl, J. A. PERK and GCN2 contribute to elF2alpha phosphorylation and cell cycle arrest after activation of the unfolded protein response pathway. Mol. Biol. Cell 16, 5493-5501 (2005).

39. Zinszner, $\mathrm{H}$. et al. $\mathrm{CHOP}$ is implicated in programmed cell death in response to impaired function of the endoplasmic reticulum. Genes Dev. 12, 982-995 (1998).

40. Han, J. et al. ER-stress-induced transcriptional regulation increases protein synthesis leading to cell death. Nat. Cell Biol. 15, 481-490 (2013).

41. Szegezdi, E., Logue, S. E., Gorman, A. M. \& Samali, A. Mediators of endoplasmic reticulum stress-induced apoptosis. EMBO Rep. 7, 880-885 (2006).

42. Malzer, E. et al. Impaired tissue growth is mediated by checkpoint kinase 1 (CHK1) in the integrated stress response. J. Cell Sci. 123, 2892-2900 (2010).

43. Thomas, S. E. et al. p53 and translation attenuation regulate distinct cell cycle checkpoints during endoplasmic reticulum (ER) stress. J. Biol. Chem. 288, 7606-7617 (2013).

44. Lin, J. H., Li, H., Zhang, Y., Ron, D. \& Walter, P. Divergent effects of PERK and IRE1 signaling on cell viability. PloS ONE 4, e4170 (2009).

45. Han, X. et al. Explore on the effect of ATF6 on cell growth and apoptosis in cartilage development. Histochem. Cell Biol. 142, 497-509 (2014).

46. Morishima, N., Nakanishi, K. \& Nakano, A. Activating transcription factor-6 (ATF6) mediates apoptosis with reduction of myeloid cell leukemia sequence 1 (Mcl-1) protein via induction of WW domain binding protein 1. J. Biol. Chem. 286, 35227-35235 (2011).

47. Luo, B. \& Lee, A. S. The critical roles of endoplasmic reticulum chaperones and unfolded protein response in tumorigenesis and anticancer therapies. Oncogene 32, 805-818 (2013)

48. Romero-Ramirez, L. et al. XBP1 is essential for survival under hypoxic conditions and is required for tumor growth. Cancer Res. 64, 5943-5947 (2004).

49. Shoulders, M. D. et al. Stress-independent activation of XBP1s and/or ATF6 reveals three functionally diverse ER proteostasis environments. Cell Rep. 3, 1279-1292 (2013).

50. Kaser, A. et al. XBP1 links ER stress to intestinal inflammation and confers genetic risk for human inflammatory bowel disease. Cell 134, 743-756 (2008).

51. Harding, H. P., Zhang, Y., Bertolotti, A., Zeng, H. \& Ron, D. Perk is essential for translational regulation and cell survival during the unfolded protein response. Mol. Cell 5, 897-904 (2000)

52. Tay, K. H. et al. Sustained IRE1 and ATF6 signaling is important for survival of melanoma cells undergoing ER stress. Cell Signal. 26, 287-294 (2014).

53. Yamamoto, K. et al. Transcriptional induction of mammalian ER quality control proteins is mediated by single or combined action of ATF6alpha and XBP1. Dev. Cell 13, 365-376 (2007) 